1 2 3

\title{
Survivability analysis of tape-tether against two concurring impacts with debris
}

\author{
Ricardo García-Pelayo ${ }^{\mathrm{a}, *}$, Shaker Bayajid Khan ${ }^{\mathrm{a}}$, Juan R. Sanmartin ${ }^{\mathrm{a}}$ \\ ${ }^{a}$ E.T.S. de Ingenieros Aeronáuticos, Universidad Politécnica de Madrid, 28040 Madrid, Spain
}

\begin{abstract}
It has recently been shown that a thin-tape tether, as opposite to a round one, has a high probability of survival to single impacts by space debris, under a broad range of de-orbit operation conditions. The purpose of the present work is to extend that analysis to survival to multiple impacts by smaller, but more abundant, debris. The method used here consist, essentially, in separating the particles into "large" and "small" ones. The large ones are so rare that the probability of them concurring on the same spot can be neglected. The small ones are a sort of background, and it is shown that the probability of them impinging close enough to a large particle crater to cause malfunction of the tape is negligible. A particular mission is considered, de-orbiting a 3,000 kg spacecraft from $800 \mathrm{~km}$ altitude at $90^{\circ}$ inclination by means of an aluminium tape of dimensions $10,000 \mathrm{~m} \times 0.06 \mathrm{~m} \times\left(5 \times 10^{-5}\right) \mathrm{m}$. It is shown that the probability that this mission survives to multiple impacts is at least 0.978 . The application of this method to missions of different parameters is also discussed.
\end{abstract}

\section{Introduction}

A successful operation of an Electrodynamic Tether system to de-orbit dead satellites necessitates the survival of the tether, which is particularly vulnerable to particle impacts because it is very long and thin. These particles can be Micrometeoroids and Orbital Debris (MMOD). Popular models ORDEM (Liou et al., 2002) and MASTER (Flegel et al., 2009) by NASA and ESA respectively, provide their flux in number of particles per year per square meter, as a function of the particle diameter, $\delta$. Of these two sources we use ORDEM, which is the most conservative of the two (that is, its flux estimation is greater than the one provided by MASTER). The M/OD population responsible for tether failure can roughly be classified in three groups: very large objects (1 m or larger), objects with diameter $(\delta)$ ranging from some $10^{-5} \mathrm{~m}<\delta<1 \mathrm{~m}$ (largely comprised of debris), and finally objects in the size range $10^{-8} \mathrm{~m}<\delta<10^{-5} \mathrm{~m}$ (largely comprised of micrometeoroids). Particles of diameter smaller than $10^{-5} \mathrm{~m}$ are too small to cause significant damage to a tape tether (Hörz, 2012).

${ }^{*}$ Corresponding author

Email addresses: r.garcia-pelayo@upm.es (Ricardo García-Pelayo), bayajid.khan@upm.es (Shaker Bayajid Khan), juanr.sanmartin@upm.es (Juan R. Sanmartin) 
A number of tether missions have been carried out in space with survivability issue yet inconclusive. Two major experimental data on the survivability of tethers in space show contrasting results (Cosmo and Lorenzini, 1997; NRO, 1996; Carroll and Oldson, 1995). One of them lasted only a few days while the other one has been orbiting for over 10 years. Conventional wisdom is that something unusual happened to the first one. Even if that is not true these two tethers have lasted an average of five years, which is about an order of magnitude more than the expected time of a typical de-orbiting tether mission (Sanmartín et al., 2015).

From a theoretical point of view it has already been shown that the probability of a tape tether - as opposed to a round tether - to be cut by a single impact is low (Khan and Sanmartín, 2013, 2014). In this article we take the next step, which is to study the probability of a tape tether to be cut by two or more concurring impacts. We show here that this probability is much smaller than the probability of being cut by a single impact. To the best of our knowledge, a quantitative investigation of the survivability of a tether with respect to concurring multiple impacts has never been done before.

In section 2 the distribution of speeds incident on the tape is derived, which is needed for later developments. In section 3 the model and its assumptions are explained. The method used in this article divides the particles into "large" and "small" and it will be applied to a tape which is $6 \mathrm{~cm}$ wide. In section 4 the probability that the larger particles do not overlap is computed. In section 5 it is shown that the probability that the smaller particles cause malfunction of the tape is negligible. In section 6 the reasons why the assumptions done in this work are conservative are listed, the extension of the computations done here to other cases is discussed and, in particular, the calculations are repeated for a tape which is $2 \mathrm{~cm}$ wide.

\section{Distribution of speeds incident on the tape}

Debris in low earth orbit (LEO) is considered to be mostly in circular orbits (Klinkrad, 2006). For high velocities $(>2 \mathrm{~km} / \mathrm{s})$, debris velocities in the horizontal plane are highly dominant (Kessler et al., 1989). In fact, debris population model ORDEM2000 (Liou et al., 2002) neglects debris radial velocity altogether and considers it to be in circular orbits.

The debris is not isotropically distributed among all inclinations (see Fig. 1). Its distribution among inclinations mimics the distribution of inclinations among satellites: near polar prograde orbits are the most frequent (Klinkrad, 2006). In this article we take as an example a tape tether in an orbit of $90^{\circ}$ inclination and take the total flux incident on it from the ORDEM2000 (Liou et al., 2002) data for $90^{\circ}$ inclination.

The distribution of the speed (relative to the tape) of the impinging debris is an essential input of the model presented in this article. In order to find it we need to know how the angle between the orbits of the debris and the tether is distributed. If the inclinations and longitudes of the ascending nodes of the tether and the debris are $i_{0}, i, \Omega_{0}$ and $\Omega$ (see Fig. 2), respectively, then the angle $\kappa$ between the orbital planes is given by the following spherical geometry formula:

$$
\cos \kappa=\cos i \cos i_{0}+\sin i \sin i_{0} \cos \left(\Omega-\Omega_{0}\right)
$$




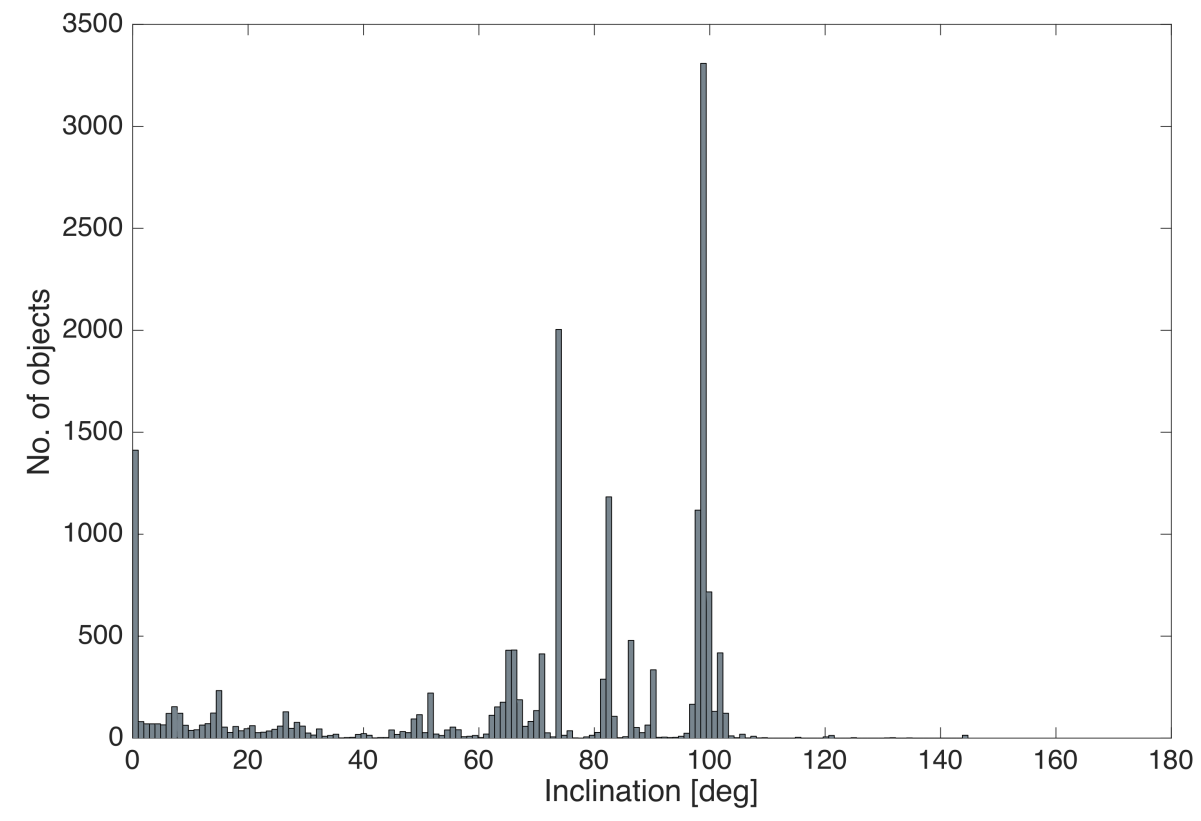

Figure 1: Distribution of inclinations of the debris. The data are from www.space-track.org.

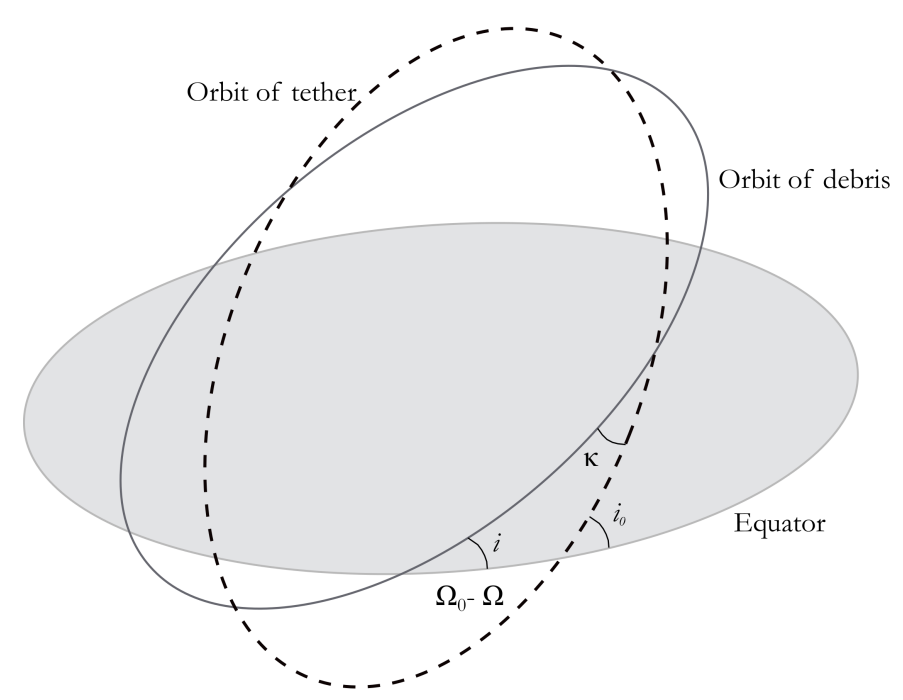

Figure 2: Relation between the angle $\kappa$ formed by the orbital planes of the tether and the debris and their inclinations and longitudes of the ascending nodes.

This formula simplifies to

$$
\cos \kappa=\sin i \cos \left(\Omega-\Omega_{0}\right)
$$

for $i_{0}=\pi / 2$. As stated above, most of the orbits have near polar inclination; to be precise, for $84.3 \%$ of them $\sin i>0.9$ (see Fig. 3 ), therefore, we may approximate the formula by 


$$
\cos \kappa \approx \cos \left(\Omega-\Omega_{0}\right),
$$

which implies that $\kappa$ is approximately distributed as $\Omega-\Omega_{0}$, which is uniformly distributed, because after one turn the orbit's precession changes $\Omega$ by an angle which, generically, is not rationally related to $2 \pi$.

There are two issues with this approximation. One is that the quantity $\sin i$ in Eq. (2) is not exactly 1 . It is a random quantity close to 1 but has a smaller average. One can check that $\kappa=\arccos \left(\sin i \cos \left(\Omega-\Omega_{0}\right)\right)$ is not exactly uniform in $[0, \pi]$ but is overrepresented around $\kappa=\arccos ( \pm \sin i)$ and underrepresented around $\kappa=\pi / 2$. This implies that the actual distribution of speeds is closer to a combination of a uniform distribution and two Dirac deltas centered at $\kappa=0$ and $\kappa=\pi$. In section 6 there is table which shows that if the calculations done for a uniform distribution of $\kappa$ are repeated for the distribution $(1 / 2)(\delta(\kappa)+\delta(\kappa-\pi))$, the results are very similar. The second, and more important,

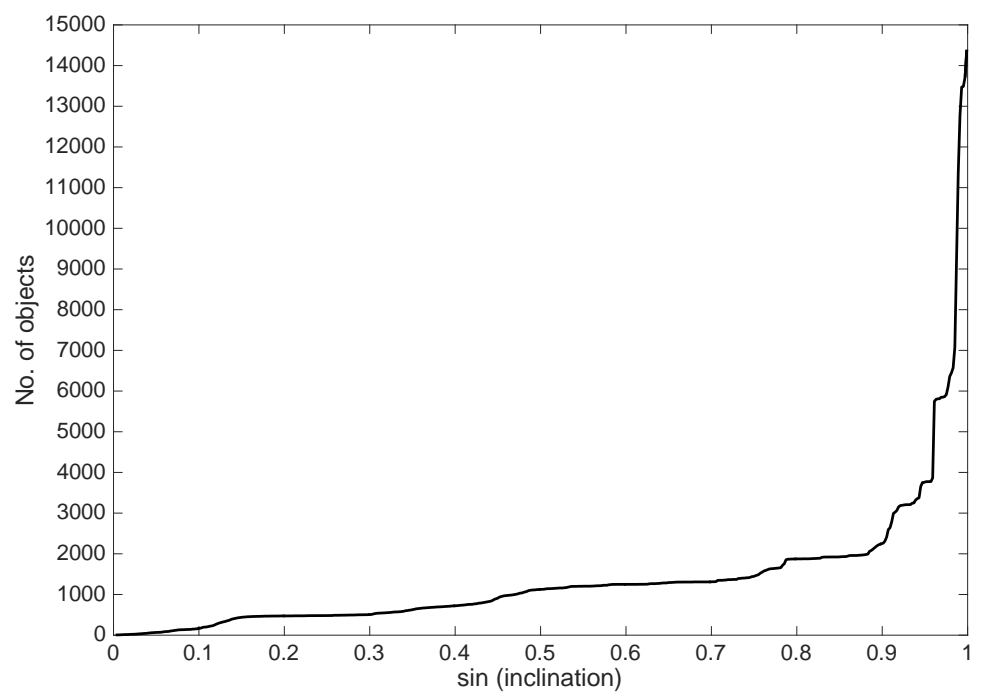

Figure 3: Cumulative distribution of the sine of the inclination of LEO debris. The data are from www.space-track.org.

issue is whether the results obtained for a tether whose orbit has a $90^{\circ}$ inclination, which simplifies Eqs. (1) to (2), are similar to the results obtained for a tether in some other orbit. For a tether orbit of inclination different from $90^{\circ}$ it would be difficult to guess an approximation to the distribution of $\kappa$. But we have repeated the calculation for a sine distribution and for the distribution $\delta(\kappa-(\pi / 2))$. These very different four distributions of speeds, shown in Fig. 4, lead to very similar results, as shown in the table of section 6. Thus we conclude that the survivability of the tape is almost independent of the distribution of speeds.

We have chosen the uniform distribution for two reasons. It is a fair approximation to the case of a polar orbit. It is simple, but not so simple that some features of the calculation for a generic distribution are absent, as is the case for the two distributions made of Dirac deltas. 


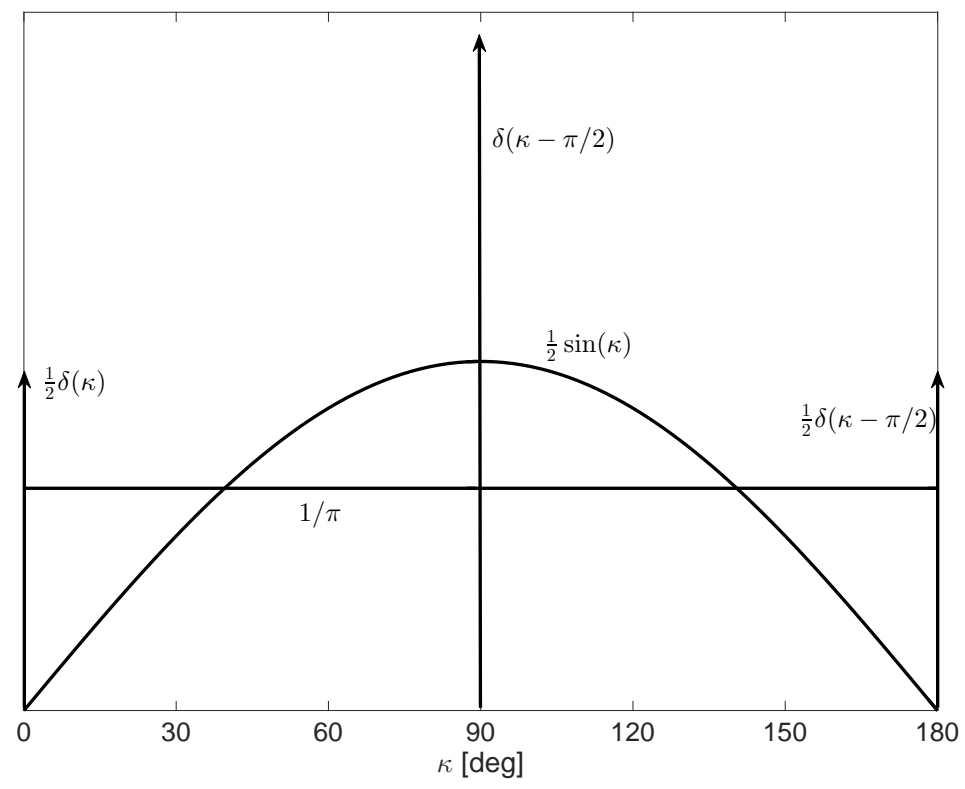

Figure 4: Four different distributions. The Dirac deltas, of course, can only be represented symbolically. The two Dirac deltas at the extremes go together, they constitute the pdf (probability density function) $(1 / 2)(\delta(\kappa)+\delta(\kappa-\pi))$. The central Dirac delta, $\delta(\kappa-(\pi / 2))$, is a different pdf.

Let there be a tether which moves in a given circular LEO of inclination $i_{0}$. Its speed is $v_{0}$, typically $7 \mathrm{~km} / \mathrm{s}$. Let $i$ be the inclination of the debris' orbit. Then the speed of that debris relative to the tape tether is

$$
v_{r e l}=2 v_{0} \sin \frac{\kappa}{2}
$$

as shown in Fig. 6.

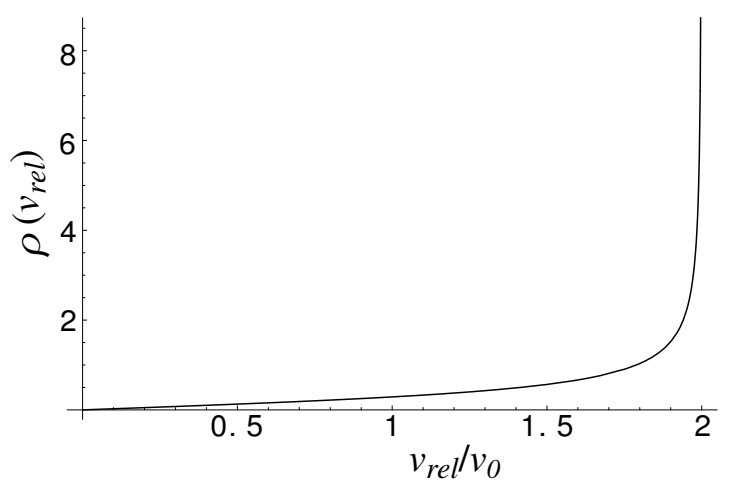

It follows from general kinetic theory (Pathria and Beale, 1972) that the pdf $\rho_{v}$ of the speed incident on the tape at $v_{r e l}$ is proportional to the flux of particles with relative speed $v_{r e l}$ and to $v_{\text {rel }}$ itself. Since, by assumption, $\kappa$ is uniformly distributed,

$$
\rho_{v}\left(v_{r e l}\right) \propto v_{r e l}\left|\frac{d \kappa}{d v_{r e l}}\right|=\frac{v_{r e l}}{v_{0} \cos \kappa / 2} .
$$

Figure 5: Pdf of the speed when all orbit inclinations are equally likely and $v_{0}=1$.
Equation (4) and normalization yield

$$
\rho\left(v_{r e l}\right)=\frac{v_{r e l}}{2 v_{0} \sqrt{4 v_{0}^{2}-v_{r e l}^{2}}}
$$


which is depicted in Fig. 5 for $v_{0}=1$.

The average speed is:

$$
\int_{0}^{2 v_{0}} d v_{r e l} \rho\left(v_{r e l}\right) v_{r e l}=\frac{\pi v_{0}}{2} .
$$
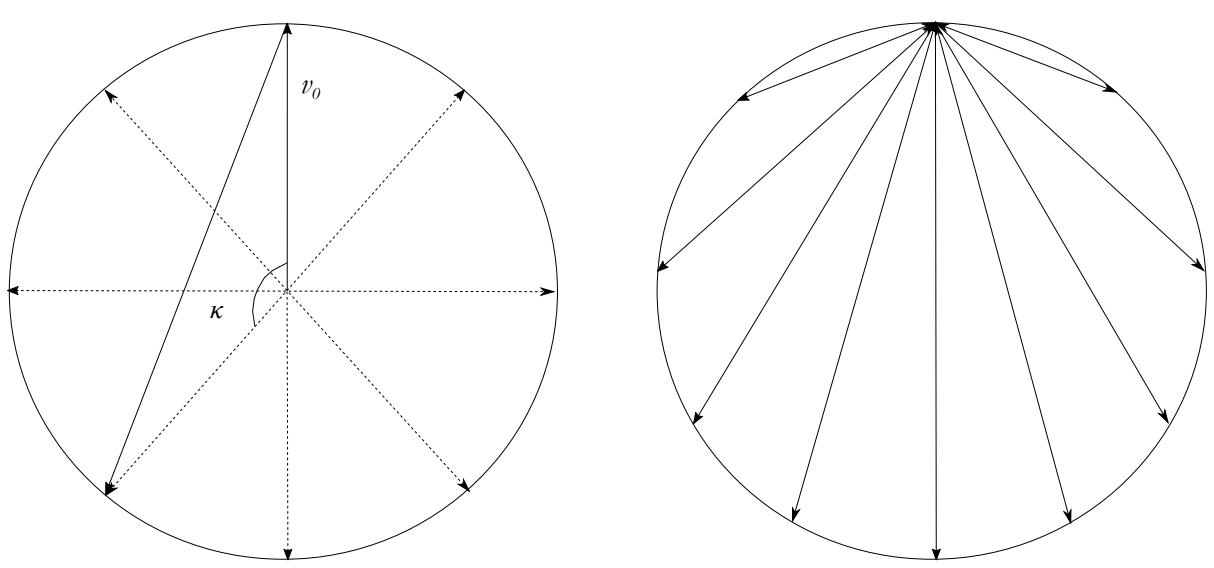

Figure 6: $\vec{v}_{0}$ is the circular velocity of the tape, which points upwardly. The circular velocities of the debris of modulus $v_{0}$ are, by assumption, isotropically distributed on the horizontal plane. They are represented by dotted vectors. The velocities of the debris relative to the tape are the velocities of the debris minus the velocity of the tape. One of them is depicted in the left figure and all others are depicted in the right Figure. Note that their component along the direction of the tape always points in the direction opposite to the tape's motion.

\section{The model}

\subsection{Mission and tape}

We consider a representative, critical mission, de-orbiting a $M_{S C}=3,000 \mathrm{~kg}$ spacecraft (SC) from $H_{0}=800 \mathrm{~km}$ altitude, at $i=90^{\circ}$ inclination. To reduce complexity in calculating probabilities, the actual de-orbit operation is skipped, assuming that the flux corresponds to the altitude of $800 \mathrm{~km}$ all the time. The duration about 3 months is taken from recent results in (Sanmartín et al., 2015), giving de-orbit time $t_{f}$ from the equation

$$
t_{f} \times\left(m_{t} / M_{S C}\right) \approx 0.174 \text { months } \times \tau\left(H_{0}, i, L / h^{2 / 3}\right) .
$$

For de-orbiting from a somewhat lower altitude, $720 \mathrm{~km}$ at $92^{\circ}$ (satellite Cryosat), Eq. (8) readily yields $t_{f} \approx 2.8$ months for a $81 \mathrm{~kg}$ aluminium tape with length $L=10 \mathrm{~km}$, width $w=6 \mathrm{~cm}$, and thickness $h=50 \mu \mathrm{m}$. When average solar-cycle ambient conditions are used to evaluate the dimensionless function $\tau$ above, this yields $\tau \approx 0.43$. The altitude $800 \mathrm{~km}$ could require more than 3 months, but this is somehow balanced in calculating probability of impacts by our keeping the flux equal to the one at $800 \mathrm{~km}$ altitude over those 3 months.

The gravity-gradient tensile force keeping a tether taut along the vertical for a spacecraft in circular orbit around a planet, assuming for simplicity equal masses $M_{S C} / 2$ at 
both ends and a much smaller tether mass, is $\frac{3}{4} \Omega^{2} L M_{S C}$ (Sanmartín et al., 2008). For 10 $\mathrm{km}$ and $3,000 \mathrm{~kg}$ in LEO $(\Omega \approx 2 \pi / 90$ minutes $)$ that force is $30 \mathrm{~N}$, yielding a tensile stress on the $6 \mathrm{~cm} \times 50 \mu \mathrm{m}$ cross section of $10^{7} \mathrm{~N} / \mathrm{m}^{2}$, which is well below the ultimate tensile strength, about $2 \times 10^{8} \mathrm{~N} / \mathrm{m}^{2}$, of many aluminium alloys. The tape might thus survive a cut reducing substantially its cross-section area, but such a cut would play havoc with the mission, which would require (over weeks / months following the cut) carrying currents as planned in the mission design over the extremely reduced cross-section. Mission survival to a cut should thus require keeping, say, $2 / 3$ of its cross-section, as considered in (Khan and Sanmartín, 2014). Therefore, in this article, we shall require that at any given horizontal plane, the length cut by the debris is always below $w / 3$.

\subsection{Minor and major axes of the craters}

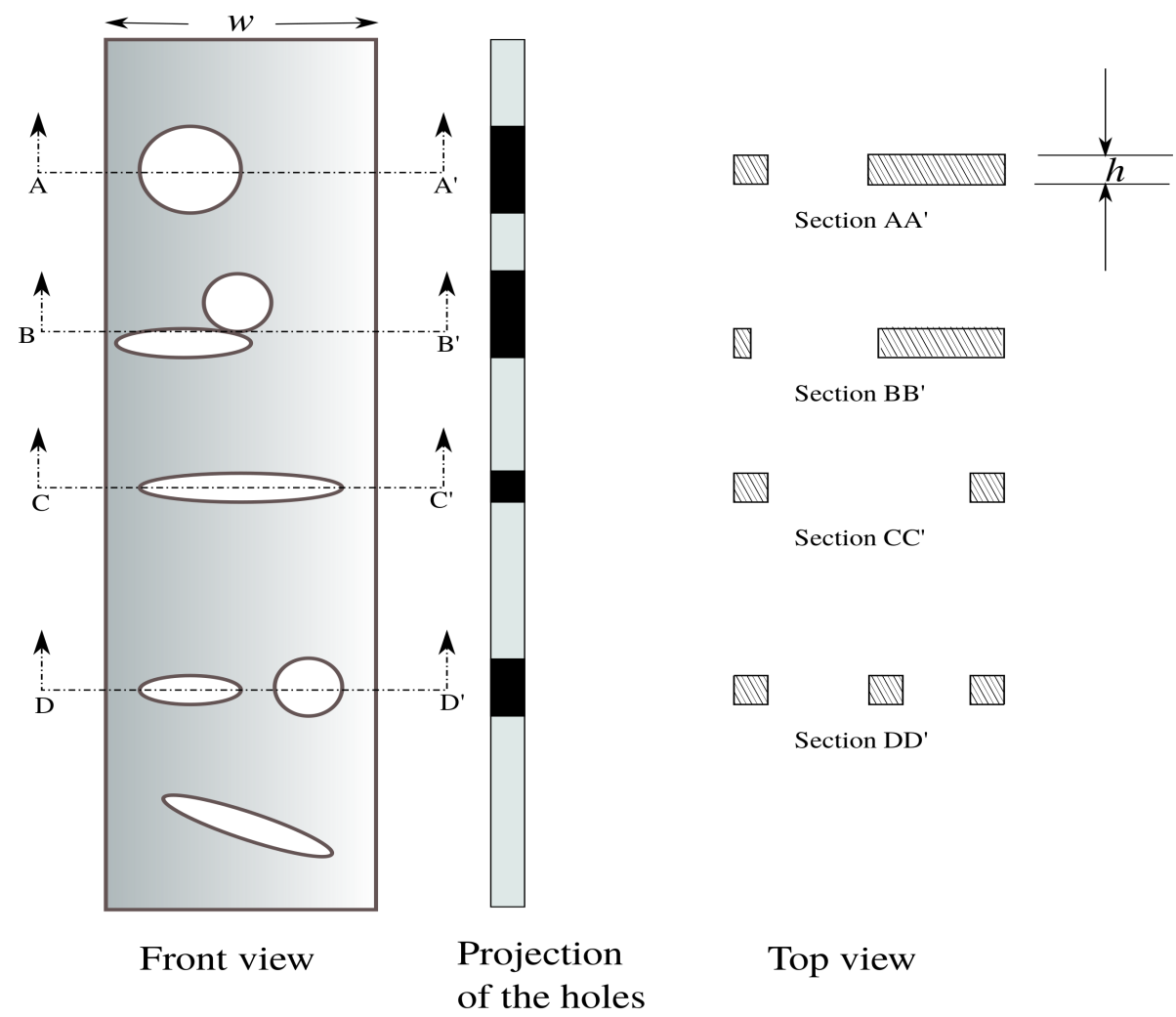

Figure 7: On the left, different damage formations and their projections onto the direction of the tape. We neglect any occurrence of damage as the last one in the bottom. On the right, sectional view from top. $h$ is the thickness of the tape and $w$ is its width.

As explained in section 2, in this article we assume that the tape, which is vertically deployed (respect to Earth) due to the gravity gradient, encounters debris particles only in the horizontal direction, as shown in Fig. 7. When a big enough particle impinges frontally on the tape, it bores a circular hole in it. If the incidence angle is different from zero, then it bores a hole of elliptical section with horizontal major axis, as shown in Fig. 7 . 
Damage equations derived from experimental data are often used to determine characteristics of impact damage and perforation (Cour-Palais, 1987; Lambert, 1997). In this article we are going to use a formula (Francesconi et al., 2013) which gives the length of the largest axis of the ellipse, $\ell$ (in $\mathrm{mm}$ ), as a function of the incidence angle, $\theta$, the diameter of the impinging particle, $\delta$ (in $\mathrm{mm})$, and the incident speed, $v_{r e l}(\mathrm{in} \mathrm{km} / \mathrm{s})$ :

$$
\ell=0.45 \delta\left(\frac{v_{r e l}}{\cos \theta}\right)^{0.65} .
$$

This formula summarizes experiments done with particles of diameter between 1 and 2 $\mathrm{mm}$ and with speeds between 3.5 and $4.6 \mathrm{~km} / \mathrm{s}$ on an aluminium tape of the same kind and thickness as the one described at the beginning of this section. The above formula holds only when the incidence angle is smaller than $80^{\circ}$. The incidence angle $\theta$ (see Fig. 8 ) is uniformly distributed. This has nothing to do with the angle $\kappa$ between the velocities of the tape and the tether. The said uniform distribution follows from the fact that the tape is twisted and has a random orientation with respect to the vertical.

For the calculations to come we need the average factor by which the vertical and horizontal axes are greater than the particle's diameter. To this end we are going to use Eq. (9). As said above, Eq. (9) has been tested only for $\theta<80^{\circ}$. But this is not a problem, because other experiments (Christiansen et al., 1993; Francesconi et al., 2013) have shown that for speeds smaller than $15 \mathrm{~km} / \mathrm{s}$ the worst incidence angle is $\theta=80^{\circ}$, because the impact damage diminishes for larger incidence angles.

In order to estimate the average horizontal axis of the hole made by a particle of diameter $\delta$, we consider the angles of incidence smaller and larger than $80^{\circ}$ separately. We use the distribution $\rho\left(v_{r e l}\right)(6)$ and Eq. (9):

$$
\begin{aligned}
0.45 \times \delta \times & {\left[\frac{80}{90} \frac{90}{80} \frac{2}{\pi} \int_{0}^{(80 / 90)(\pi / 2)} d \theta \int_{0}^{2 v_{0}} d v_{r e l} \rho\left(v_{r e l}\right)\left(\frac{v_{r e l}}{\cos \theta}\right)^{0.65}+\right.} \\
& \left.\frac{10}{90} \int_{0}^{2 v_{0}} d v_{r e l} \rho\left(v_{r e l}\right)\left(\frac{v_{r e l}}{\cos 80^{\circ}}\right)^{0.65}\right] \approx 3.56 \delta .
\end{aligned}
$$

This estimation is actually an upper bound because for angles of incidence between $80^{\circ}$ and $90^{\circ}$, for which the second term accounts, we have assumed that the major axis is the one for $80^{\circ}$, while it is actually shorter (Christiansen et al., 1993), as said above.

The average projection of the hole onto the vertical direction made by a particle of diameter $\delta$ is equal to the horizontal one only when the incidence angle is $0^{\circ}$, that is:

$$
0.45 \times \delta \int_{0}^{2 v_{0}} d v_{r e l} \rho\left(v_{r e l}\right)\left(\frac{v_{r e l}}{\cos 0}\right)^{0.65} \approx 2.21 \delta .
$$

\subsection{Outline of the calculation}

The numbers appearing in this subsection will be obtained in sections 4 and 5 .

We shall see that the probability that the craters bored by particles larger than 1 $\mathrm{mm}$ overlap is about 0.0054 . Therefore we may picture, with $(1-0.0054)$ certainty, that the tape has a number of non overlapping craters due to "large" particles and a 

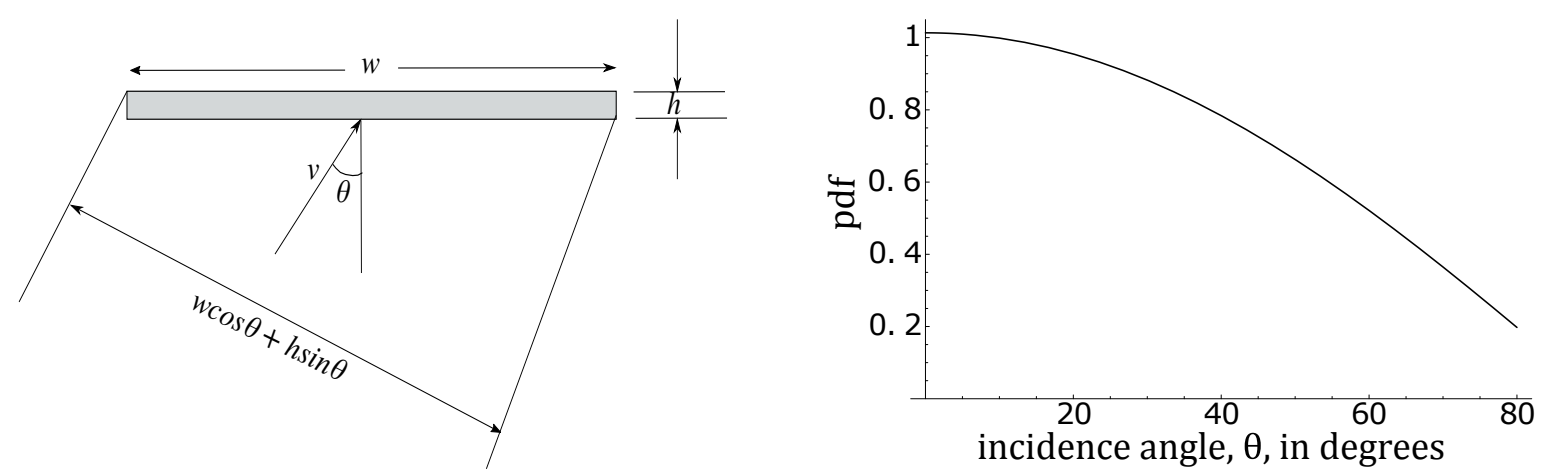

Figure 8: As shown on the left of the Figure the probability that the incidence angle is $\theta$ carries a geometrical proportionality factor of $w \cos \theta+h \sin \theta$. Upon normalization yields this yields the pdf $\frac{w \cos \theta+h \sin \theta}{h-h \cos 80^{\circ}+w \sin 80^{\circ}}$, which is graphed on the right.

background of craters caused by small particles. We define the cell associated to a crater as the rectangle on the tape which is delimited by the vertical edges of the tape and the horizontal segments tangent to the crater, as depicted in Fig. 9. A straightforward calculation based on the flux versus diameter data yields that the average additional length that the smaller particles cut in the cell associated to the crater bored by a large particle is of the order of $10^{-2} \mathrm{~mm}$. Therefore only an unlucky concentration of small particles in a cell could cut additional tape so that the total length cut amounted to $w / 3$.

For our tape, the probability that there are more than 5 single impacts which cut more than $1 \mathrm{~cm}$ of tape each is 0.0015 . We shall show that the probability that the smaller particles add more than $3 \mathrm{~mm}$ to any of these 4 single impacts is 0.0157 . Since $10 \mathrm{~mm}+3 \mathrm{~mm}$ is still below $w / 3=2 \mathrm{~cm}$, it follows that, with a probability of about $(1-0.0054)(1-0.0015)(1-0.0157)=0.978$ multiple impacts do not add significant threat to single impacts.

This argument will be rephrased with greater generality in section 6 , and a comment on its validity will be made.

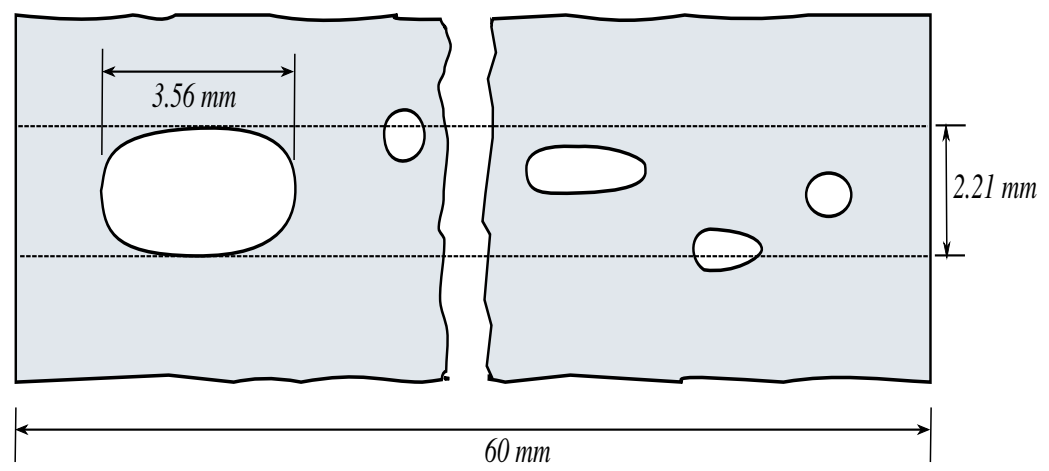

Figure 9: The cell corresponding to a crater bored by a particle of $1 \mathrm{~mm}$ diameter is shown. The maximum length cut by the smaller particles along any given horizontal line is less than the sum of their horizontal axes, because the smaller particles are randomly distributed in the cell. We suppose that the said maximum length is less than half of the sum of their horizontal axes. 


\section{Overlap probability of the larger particles}

The impactors can be micrometeoroides or debris of human origin. Their cumulative distributions are available (Grün et al., 1985; Liou et al., 2002). The original data are fluxes per year per $\mathrm{m}^{2}$. Since the cumulative distribution $F(\delta)$ provided by Grün et al. (1985) and Liou et al. (2002) is related to the density of flux $f(\delta)$ by

$$
F(\delta) \equiv \int_{\delta}^{\infty} d \delta^{\prime} f\left(\delta^{\prime}\right)
$$

the density of flux is minus the derivative of this cumulative distribution. As stated above we use the data corresponding to a polar orbit $\left(i=90^{\circ}\right)$ at a height of $800 \mathrm{~km}$.

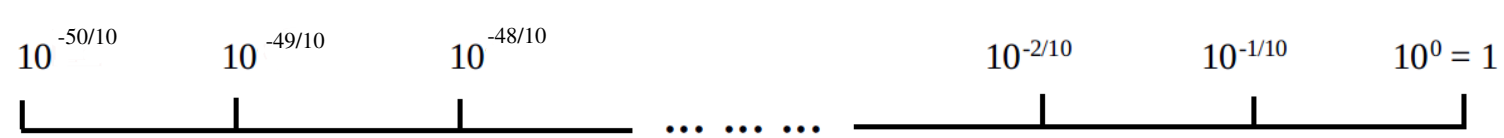

Figure 10: Bins used in this article. The units are meters.

In order to do computations, we divide the range of diameters of the particles in bins such that the size of each bin is $10^{1 / 10}$ times greater than the bin which neighbours it to the left. In other words, in a log paper each decade is divided into 10 bins of equal width. The limits between neighbouring bins are inverse powers of ten times the numbers $\left\{10^{1 / 10}, 10^{2 / 10}, \cdots, 10^{9 / 10}, 10^{10 / 10}\right\} \approx\{1.26,1.58,2,2.51,3.16,3.98,5.01,6.31,7.94,10\}$. In particular they are the numbers $10^{j / 10}$, where $j$ is a negative integer. In this article the diameter of a particle in an interval will always be set equal to the upper limit of the interval, in order to overestimate the damaged length.

As advertised in section 3, in this section we are interested in particles larger than 1 $\mathrm{mm}$, therefore in this calculation $j$ will range from -30 to 0 , because $10^{-(30 / 10)} \mathrm{mm}=1$ $\mathrm{mm}$. We are going to need the pdf $f$ of the diameters of the impinging particles. $f$ will be approximated by

$$
\begin{gathered}
f(\delta) \approx-\frac{F\left(10^{(i-1) / 10}\right)-F\left(10^{i / 10}\right)}{\left(F\left(10^{-30 / 10}\right)-F\left(10^{0 / 10}\right)\right)\left(10^{(i-1) / 10}-10^{i / 10}\right)}, \\
\quad \text { for } \delta \in\left[10^{(i-1) / 10}, 10^{i / 10}\right], \quad i=-29, \cdots, 0 .
\end{gathered}
$$

$F\left(10^{0 / 10}\right) \approx 0$, but it was included in the denominator above for the sake of mathematical clarity. We recall that the average minor (vertical) axis of the crater is 2.21 times the diameter of the impinging particle (11). In order to compute the probability $p_{n o}$ that the projections of the minor axes of the holes onto the direction of the tape do not overlap we use the formula

$$
p_{n o}(L, \varphi, n)=\left(\frac{L-n \bar{\ell}}{\exp \int d \ell \varphi(\ell) \ln (L-\ell)}\right)^{n}=\left(\frac{L-n \bar{\ell}}{\exp \overline{\ln (L-\ell)}}\right)^{n},
$$


which is derived in the Appendix. In it $\bar{\ell}$ is the average length of the minor axis of the holes whose minor axis is larger than $2.21 \mathrm{~mm}, L$ is the length of the tape, $\varphi$ is the pdf of the vertical axes which are greater than $2.21 \mathrm{~mm}$ and $n$ is the total number of impacts by particles larger than $1 \mathrm{~mm}$ on both sides of the tape during its mission. The relation between $\varphi$ and $f$ is

$$
\varphi(\ell)=\frac{1}{2.21} f\left(\frac{\ell}{2.21}\right) .
$$

In SI units, $L=10000, \bar{\ell}=0.0028, n=139.3$ and $\exp \overline{\ln (L-\ell)} \approx 10000$, from which:

$$
p_{\text {no }}(10000, \varphi, 139.3) \approx 0.9946=1-0.0054,
$$

as advertised in subsection 3.3.

\section{The smaller particles}

In order to study the threat posed by the smaller particles, we are going to study the additional length which they might cut in the cells that correspond to the craters bored by the larger particles. We consider cells whose height ranges from $2.21 \mathrm{~mm}$ (bored by particles of $1 \mathrm{~mm}$ diameter) to $12.4 \mathrm{~mm}$. Cells of height larger than $2.21 \times(20 / 3.56) \mathrm{mm}$ $=12.4 \mathrm{~mm}$, correspond, as follows from Eqs. (10) and (11), to particles which, on the average, cut $1 / 3$ of the tape and disable it. This is the problem of cut by a single particles which has been addressed already (Khan 2014).

The average length which the smaller particles can cut in one of the largest cells can be computed by multiplying the expected number of impacts on a $60 \times 12.4 \mathrm{~mm}^{2}$ in three months for each size bin by the $\delta$ of that bin and then multiplying this number by 3.56 (see Eq. (10)). This yields $0.06 \mathrm{~mm}$. This is the sum of the major axes of the craters. The maximum length that they may cut along any given horizontal direction is of course less, probably less than half (see Fig. 9), which would be about $0.03 \mathrm{~mm}$. This quantity is negligible, but, as stated above, the smaller particles might concentrate in the cell of the crater of one of the large particles and cut additional tape so that the total length cut amounts to $2 \mathrm{~cm}$, which is $1 / 3$ of the tape's width.

The number of craters which are dangerously big is very small. The probability of impacts boring craters whose major axis is over $1 \mathrm{~cm}$ can be read off the flux data. Using the Poisson distribution one finds that the probability that there are more than 5 such craters is 0.0015 . In order to see that the probability that significant length is cut in any of these 5 cells by the smaller particles is negligible, we have to study the fluctuations in the number of impacts of the small particles on a rectangle.

The Poisson distribution applied to this problem is

$$
P(n)=e^{-\bar{n}} \frac{\bar{n}^{n}}{n !},
$$

where $\bar{n}$ is the mean number of particles that impinge on a cell in three months. We may write 


$$
\bar{n}=\sum_{i=-49}^{0} \bar{n}(i)
$$

where

$$
\bar{n}(i) \equiv F\left(10^{(i-1) / 10}\right)-F\left(10^{i / 10}\right),
$$

is the mean number of impacts by particles in the $i$-th diameter range in three months on a cell. $F$ is now the flux per rectangle and per three months. Thus there is a Poisson distribution for each diameter bin, with mean $\bar{n}(i)$.

For the $i$-th bin defined in section 4 (see Fig. 10), we define a number of particles (per little rectangle, per three months) $n(p, i)$ such that the probability that the actual number of impacts by particles in the $i$-th bin be greater than $n(p, i)$ is at most $p$. To be precise, we define $n(p, i)$ as the smallest integer satisfying the inequality

$$
e^{-\bar{n}(i)} \sum_{n=n(p, i)+1}^{\infty} \frac{\bar{n}(i)^{n}}{n !} \leq p .
$$

The $n(p, i)$ 's can be found numerically for each bin. Clearly, when $p<<1$, the $n(p, i)$ 's are significantly (much) larger than $\bar{n}(i)$. The idea exploited in this section is to choose values $n(p, i)$ high enough so that the probability of surpassing them is negligible, but small enough so that the added impacted length is still acceptably small.

We choose $p=10^{-5}$. Then $\left\{n\left(10^{-5}, i\right)\right\}_{i=-50}^{-31}=\{2,3,3,3,3,3,2,2,2,2,2,2,2,1,1,1,1$, $1,1,1\}$. We use this result as an initial guess and use $\left\{n_{i}^{\prime}\right\}_{i=-50}^{-31}=\{2,3,3,3,3,3,2,2,2,2,2$, $2,1,1,1,1,0,0,0,0\}$ instead. The probability that in a given rectangle $n_{i}^{\prime}$ is not surpassed is

$$
e^{-\bar{n}(i)} \sum_{n=0}^{n_{i}^{\prime}} \frac{\bar{n}(i)^{n}}{n !}
$$

When the width of the rectangle is $12.4 \mathrm{~mm}$, the probability that the number of particles of diameter in each $i$-th bin is kept under $n_{i}^{\prime}+1$ in all five cells is

$$
\left(\prod_{j=-50}^{-31}\left(e^{-\bar{n}(j)} \sum_{m=0}^{n_{j}^{\prime}} \frac{\bar{n}(j)^{n}}{m !}\right)\right)^{5} \approx 0.9843
$$

The sum of the diameters of the craters produced by particles whose occupation numbers of the size bins are $\left\{n_{i}^{\prime}\right\}_{i=-50}^{-31}=\{2,3,3,3,3,3,2,2,2,2,2,2,1,1,1,1,0,0,0,0\}$ is

$$
3.56\left(\sum_{i=-49}^{-30} n_{i}^{\prime} 10^{i / 10}\right) \mathrm{m} \approx 7.75 \mathrm{~mm} .
$$

As mentioned above,the maximum length that cut by the smaller particles along any given horizontal direction is probably less than half the above length (see Fig. 9), say about $3 \mathrm{~mm}$. Since the centimeter cut by the large particles and the $3 \mathrm{~mm}$ cut by the small particles do not add to $w / 3=2 \mathrm{~cm}$, it follows that multiple impacts do not add 
significant threat to single impacts. To associate a probability with this statement we may multiply the probability of no overlap of the large particles found in section 4 by the two probabilities found in this section: $0.9946(1-0.0015) 0.9843 \approx 0.978$.

0.9843 is an underestimation of the probability that the sum of the diameters of the craters produced by the particles which have impacted on each of the 5 cells is smaller than $3 \mathrm{~mm}$ for 2 reasons. First, because keeping all the fluxes under $n_{i}^{\prime}+1$ is not the only way in which this event might take place. Other $\left\{n_{i}^{\prime}\right\}_{i=-50}^{-31}$ could also be compatible with this event. Second, and foremost, because we have chosen the cell of height $12.4 \mathrm{~mm}$, which is the largest in the range $[2.21 \mathrm{~mm}, 12.4 \mathrm{~mm}]$.

\section{Discussion}

\subsection{Calculations for different mission parameters}

We have computed an approximation to the probability of survival with respect to cut by concurring particles for a tape which is $10000 \mathrm{~m}$ long on a three month mission and whose orbit has a $90^{\circ}$ inclination. We would like to have an idea of how this survival probability changes when the dimensions of the tape, the duration of the mission and the inclination change.

It is very easy to see how the calculations of section 4 have to be modified because in the applications that we have in mind, $n \bar{\ell}<<L$ and the following approximation is very good:

$$
p(L, \varphi, n) \approx\left(\frac{L-n \bar{\ell}}{L}\right)^{n}=\left(1-\frac{n \bar{\ell}}{L}\right)^{n} \approx \exp -\frac{\bar{\ell}}{L} n^{2} .
$$

$n$ is the expected number of impacts, which is proportional to the duration $t$ of the mission and to the length of the tape, that is $n=$ const. $L t . \bar{\ell}$ depends on the choice of separation between small and large particles, "large particles" being the ones that don't overlap. In the example presented so far we have taken this separation to be $1 \mathrm{~mm}$. If we take the debris flux to be constant (conservatively, the largest one encountered during the mission), then $\bar{\ell}$ may be taken as a constant. Then the dependence of the probability of survival on the dimensions of the tape and the duration $t$ of the mission is well approximated by

$$
\begin{gathered}
p_{n o}(\bar{\ell} ; L, t) \approx \exp -\bar{\ell} \frac{n^{2}}{L}=\exp -\bar{\ell} \frac{\operatorname{const}^{2} w^{2} L^{2} t^{2}}{L}=\exp -\bar{\ell} \operatorname{const}^{2} w^{2} L t^{2}= \\
\exp -\bar{\ell} \operatorname{const}^{2} w_{0}^{2} L_{0} t_{0}^{2} \frac{w^{2} L t^{2}}{w_{0}^{2} L_{0} t_{0}^{2}}=p_{n o}\left(\bar{\ell} ; L_{0}, t_{0}\right)^{\frac{w^{2} L t^{2}}{w_{0}^{2} L_{0} t_{0}^{2}}}=0.9946^{\frac{w^{2} L t^{2}}{w_{0}^{2} L_{0} t_{0}^{2}}}
\end{gathered}
$$

The dependence of $p_{n o}$ on time and width is stronger than the dependence on the length. For a tape twice as long the probability $p_{n o}$ of no overlap will diminish to $p_{n o}{ }^{2}$, but if the mission lasts twice, then $p_{n o}$ will diminish to $p_{n o}{ }^{4}$. Since we have taken a extreme case, typically $p_{n o}(\bar{\ell} ; L, t)$ will be even closer to 1 .

The calculation done in section 5 is more involved, and it is not so easy to see how it scales. The area of the largest cells is about $w^{2} / 5$ (because $2.21 / 3.56 / 3 \approx 1 / 5$, see the first paragraph of section 5), and we demand that the length added by the small particles be less than about $w_{s}=3 \mathrm{~mm}$. The flux on $w^{2} / 5$ depends only on the width 
and the time, and therefore so does the the length added by the small particles. When $w$ diminishes, the average flux diminishes as $w^{2}$, but for a given $p$ the fluctuations given by $n(p, i)$ do not diminish that much, and the length which might be destroyed by small particles does not diminish that much. However, calculations similar to the ones done in section 5 for the case of a $10 \mathrm{~km} \times 2 \mathrm{~cm}$ tape yield that the smaller particles still don't pose any additional significant threat. Before showing the results, we rephrase subsection 3.3 in greater generality and introduce some notation which will be helpful to compare the calculations done for both tapes.

The probability that the craters caused by particles larger than $\delta_{o}$ overlap is $p_{0} \equiv p_{n o}<$ $10^{-2}$, therefore we assume that they do not overlap. The only problem is then if the smaller particles cut the tape in the cell of one of the large particles (see Fig. 9) and cut additional tape so that the total length cut amounts to $w / 3$. The probability that there are more than $d i$ ("dangerous impacts") impacts which cut more than $w / 6$ each is $p_{d i}<10^{-2}$. We show that the probability that the smaller particles add more than about $w_{s} \lesssim w / 10$ of cut length to any of the craters bored by the $d i$ single impacts is $p_{s} \approx 10^{-2}$. Since $w / 6+w_{s}$ is still below $w / 3$ and $1-\left(1-p_{o}\right)\left(1-p_{d i}\right)\left(1-p_{s}\right)<<1$, it follows that multiple impacts do not add significant threat to single impacts.

Strictly speaking, this argument does not preclude the possibility that some single impact cuts more than $w / 3-w_{s}$ and thus the addition of smaller craters reaches the $w / 3$ limit. This possibility, however, would destroy at most $w / 3+w_{s}$ of the tape. But the $w / 3$ limit is not strictly defined (Sanmartin...), so the tape, although not optimally, would still work because $w_{s}$ is very small. So this unlikely event does not invalidate the calculations presented here.

In the table that follows we summarize the results for the $6 \mathrm{~cm}$ tape, already seen, and for a $2 \mathrm{~cm}$ tape, all other parameters remaining the same. The only heading which has not been defined yet is $\Pi \equiv\left(1-p_{o}\right)\left(1-p_{d i}\right)\left(1-p_{\text {small }}\right)$. We saw in section 5 that for the $6 \mathrm{~cm}$ tape, $\left\{n_{i}^{\prime}\right\}_{i=-50}^{-31}=\{2,3,3,3,3,3,2,2,2,2,2,2,1,1,1,1,0,0,0,0\}$. For the 2 cm tape, $\left\{n_{i}^{\prime}\right\}_{i=-50}^{-33}=\{1,2,2,2,2,2,1,1,1,1,1,1,1,1,1,1,0,0\}$. As advertised in section 2 , for each tape three other different distributions of speeds are used, to show that the results depend very little on this choice.

The results depend also very little on the inclination of the tether's orbit. A change in the inclination entails a change in the flux and a change in the distribution of the angle $\kappa$ between the orbital planes of the tether and the debris. But we have already seen that the $800 \mathrm{~km}, 90^{\circ}$ flux is among the largest and that, as the table that follows shows, the results depend very little on the distribution of $\kappa$.

\begin{tabular}{|c|c|c|c|c|c|c|c|c|}
\hline$w(\mathrm{~cm})$ & distribution & $\delta_{0}(\mathrm{~m})$ & $p_{0}$ & $d i$ & $p_{d i}$ & $w_{s}(\mathrm{~m})$ & $p_{s}$ & $\Pi$ \\
\hline \hline 6 & extremes & 0.001 & 0.00320 & 3 & 0.00013 & 0.003 & 0.0089 & 0.988 \\
6 & uniform & 0.001 & 0.00540 & 5 & 0.00150 & 0.003 & 0.0157 & 0.978 \\
6 & sine & 0.001 & 0.00530 & 6 & 0.00040 & 0.003 & 0.0187 & 0.976 \\
6 & central & 0.001 & 0.00514 & 6 & 0.00032 & 0.004 & 0.0177 & 0.977 \\
\hline 2 & extremes & 0.00063 & 0.00455 & 2 & 0.00011 & 0.002 & 0.0006 & 0.995 \\
2 & uniform & 0.00063 & 0.00764 & 41 & 0.00118 & 0.002 & 0.0136 & 0.978 \\
2 & sine & 0.00063 & 0.00744 & 41 & 0.00024 & 0.002 & 0.0113 & 0.981 \\
2 & central & 0.00063 & 0.00723 & 41 & 0.00011 & 0.002 & 0.0113 & 0.981 \\
\hline
\end{tabular}


6.2. Why rare large debris particles are more dangerous than very abundant small debris particles

The reader familiar with the topic of this article may find it puzzling that only the largest debris matters. The reason for this puzzlement could be stated as follows: On a log-log paper the slope of the cumulative distribution of the diameter of the impactors is, except for a small region around $10^{-5} \mathrm{~m}$ and for $\delta \in\left[10^{-1} \mathrm{~m}, 1 \mathrm{~m}\right]$, always $<-1$ (actually, it is between -4 and -2.5 for most of the range, see Fig. 11), therefore the slope of the pdf $f$ is $<-2$. When this density is multiplied by the length, which is proportional to the diameter, the slope is still negative, which means that the destroyed length is a decreasing function of the diameter of the impactors. Why is it then that the impactors which matter are not the smallest, but the largest ones? The average destroyed

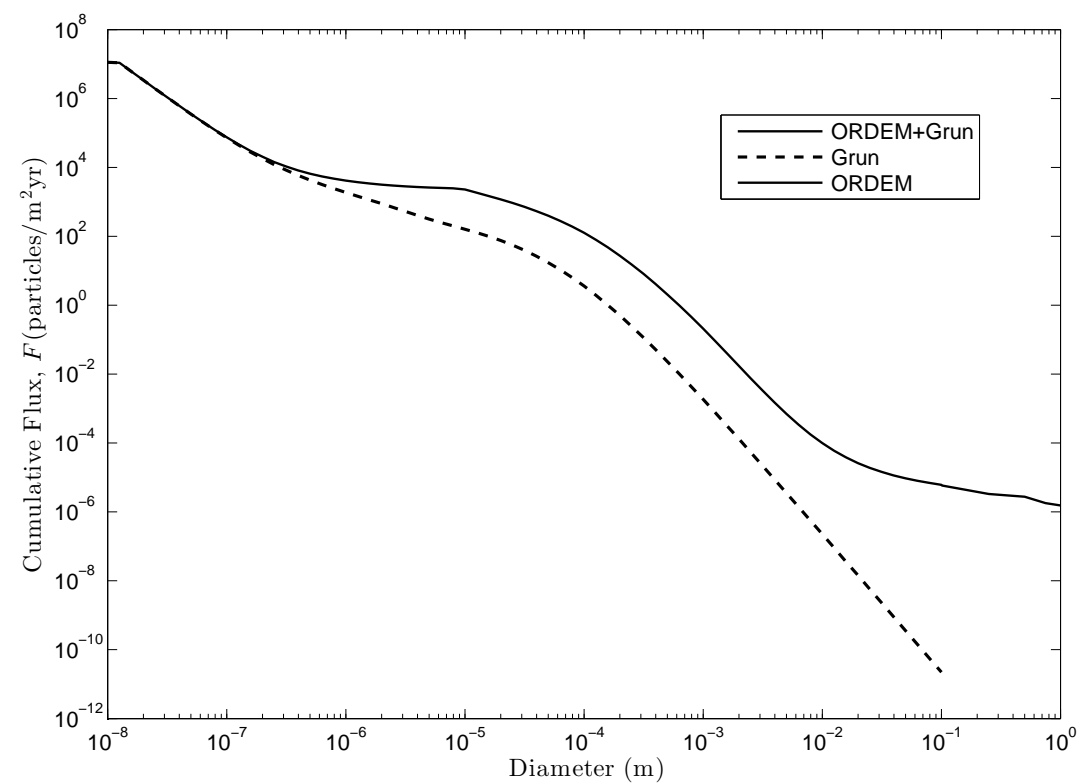

Figure 11: Flux data of a circular orbit with inclination of $90^{\circ}$ and $800 \mathrm{~km}$ altitude. The cumulative distribution of debris, ORDEM2000, is undistinguishable from the total cumulative distribution for diameters greater than $10^{-5} \mathrm{~m}$, because in that size range the flux of micrometeoroids is negligible compared to the flux of debris particles. The cumulative distribution $F$ used here is related to the density $f$ by $F(\delta) \equiv \int_{\delta}^{\infty} d \delta f(\delta)$.

length is a decreasing function of the diameter of the impactors, but the average destroyed length is negligible anyway. For more than $w / 3$ to be destroyed somewhere, we have to consider very unlikely fluctuations above the mean flux. For small particles to destroy a significant length of the tape, a fluctuation involving many small particles would be required, which is very unlikely since the Poisson distribution, for large averages, behaves as a Gaussian. However, for large particles to destroy a significant length of the tape, a fluctuation involving only a few large particles is required. There are few large particles 
and the Poisson distribution decays rather slowly for small averages (it has a "fat tail"), thus such fluctuations are much more likely than for small particles.

\subsection{Conservativeness of the assumptions}

There are a number of conservative assumptions in our model. Since this is an important aspect of it, we gather here the most important of them.

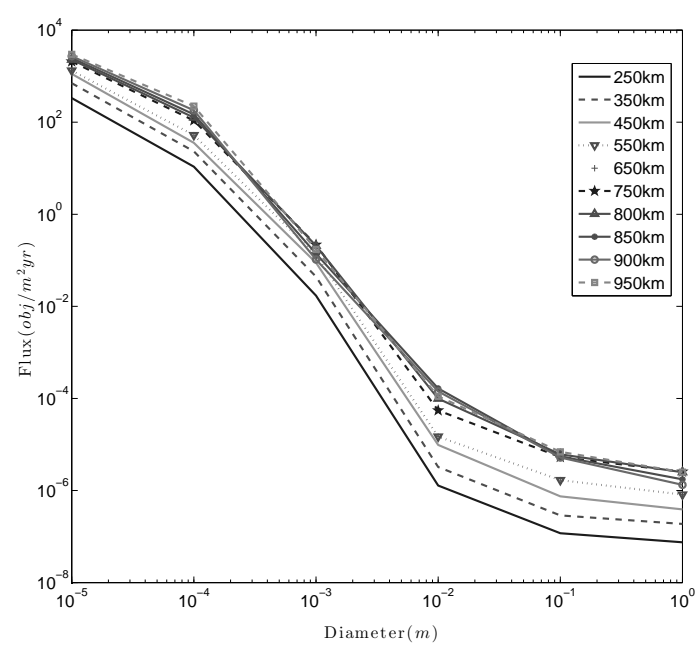

(a)

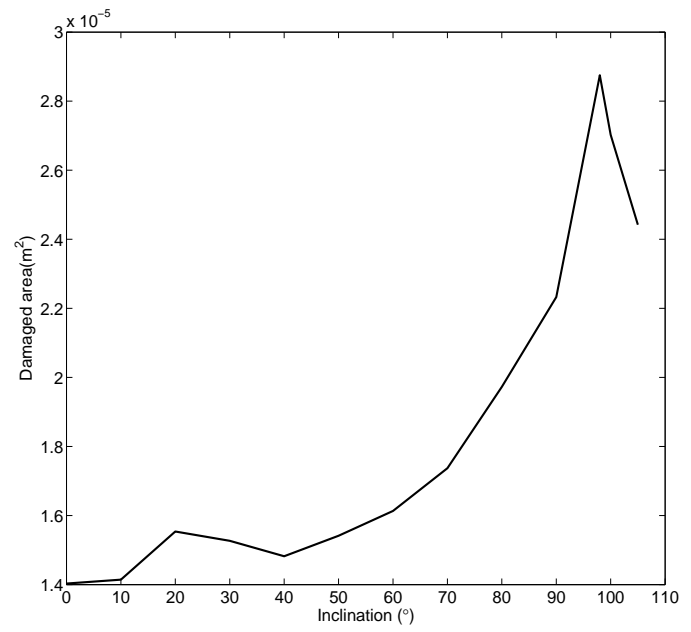

(b)

Figure 12: (a) In general the flux (at $90^{\circ}$ ) diminishes as the altitude diminishes. (b) For all the altitudes considered in Fig. 12a, the flux encountered at $90^{\circ}$ is almost the largest of all inclinations.

1) For geometrical reasons the oblique incidences are the least frequent, as explained in Figure 8. This shows that the the factor computed in Eq. (10) is actually not as big.

2) If the tether is not exactly vertical (i. e., if it is not exactly self balanced (Pelaez and Sanjurjo, 2006) then the component of the crater along the direction of the width $w$ is not as large as we have assumed in this article. See the bottom crater of Fig. 7.

The four next facts show that the fluxes used in the calculations are conservative.

3) Let $\vec{v}_{0}$ be the orbital velocity for the circular orbit that we have assumed for the tape. $v_{0}$ is of the order of $7 \mathrm{~km} / \mathrm{s}$. The speed of the incoming particles ranges between 0 if they come perpendicularly to the direction of motion of the tape, to $2 v_{0}$ if they come head on. This is shown in Figure 6. For the smallest speeds of incidence the incoming particle is not able to bore the tape.

4) We take the debris flux to be the one at $800 \mathrm{~km}$ altitude. As the hypothetical defunct satellite comes down, the flux of debris diminishes (see Fig. 12a), therefore we are being conservative when we take the flux to be the one at $800 \mathrm{~km}$ at any height. This simplifies the calculations very much.

5) The inclination of $90^{\circ}$ is second only to the $100^{\circ}$ inclination in the total impacted area, as shown in Fig. 12b

6) In the computations in section 5 we have chosen the cell of height $(w /(3 \times 3.56))$, which is the largest in the range $\left[2.21 \delta_{o}, 2.21(w /(3 \times 3.56))\right]$ 


\section{Conclusions}

This work presents a quantitative assessment of the risk of multiple impact of tape tethers in operation at LEO with debris particles. For a particular chosen mission and by separating the particles into "large" (about $1 \mathrm{~mm}$ or above) and "small", it has been shown in section 4 that the probability that holes created by large particle do not overlap is greater than 0.99 , and when smaller particles are taken into account, the overall probability to survive mission failure criteria (i.e crater length $>w / 3$ ) is about 0.98 or greater, as shown in section 5 .

Furthermore, considering a range of conservative assumptions, the calculation has been validated for a range of different tape and mission parameters to show the insignificancy of multiple impacts.

\section{Acknowledgements}

This work was supported by an Universidad Politécnica de Madrid Research Grant (RR01/2009) and by European Commission FP7 Space Project 262972. Conversations with Juan José Negrete Solana are gratefully acknowledged. 


\section{Appendix}

Let there be $n$ segments of length $\ell_{i}$ randomly placed on a long segment of length $L$, such that $\ell_{1}+\cdots+\ell_{n}<L$. Let us, for the moment, denote the position of a short segment by the coordinate of its left end. Then the position of the $i$-th segment takes a random value in $\left[0, L-\ell_{i}\right]$. Then there is a one-to-one correspondence between the possible configurations of the short segments and the points in $\left[0, L-\ell_{1}\right] \times \cdots \times\left[0, L-\ell_{n}\right]$.

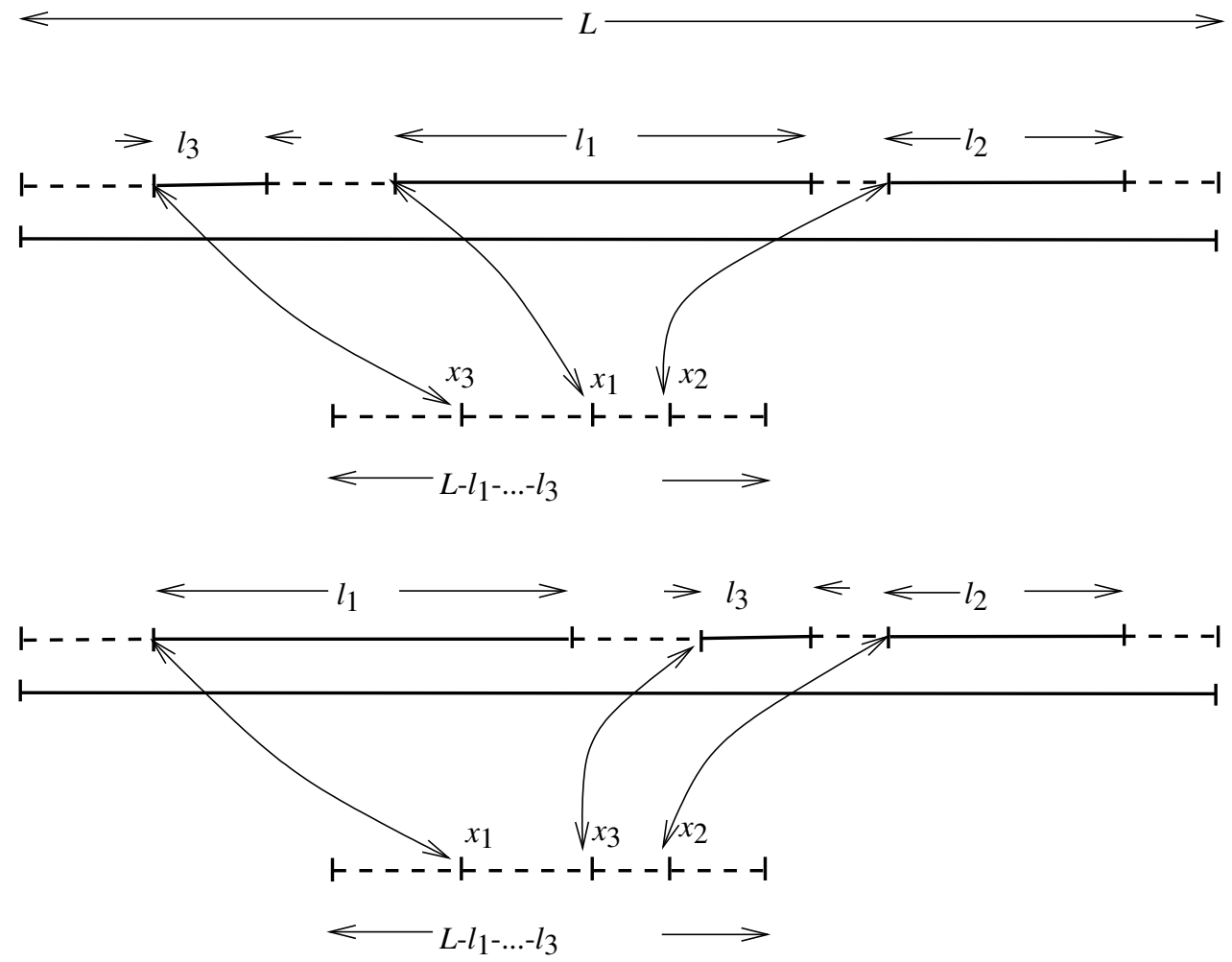

Figure 13: The space left uncovered by the short segments has been drawn dashed between the segments and collected in the lower part of the Figure. $x_{i}$ is the uncovered length of the long segment which lies to the left of the left end of the $i$-th segment. Note that the 3-tuples $\left(x_{3}, x_{1}, x_{2}\right)$ and $\left(x_{1}, x_{3}, x_{2}\right)$ correspond to different orderings of the segments.

When the short segments do not overlap they leave an uncovered space of length $L-\left(\ell_{1}+\cdots+\ell_{n}\right)$ which they partition in $n+1$ pieces. In this case we may denote the position of the $i$-th short segment by the uncovered length $x_{i}$ of the long segment which lies to the left of its left end. Then there is then a one-to-one correspondence between the non-overlapping configurations and the set of ordered $n$-tuples which take values in the hypercube $\left[0, L-\sum_{i=1}^{n} \ell_{i}\right]^{n}$, as shown in Fig. 13. It follows that the probability that a random laying of short segments on the long segment has no overlaps is

$$
p\left(L, \ell_{1}, \ldots, \ell_{n}\right)=\frac{\left(L-\sum_{i=1}^{n} \ell_{i}\right)^{n}}{\prod_{i=1}^{n}\left(L-\ell_{i}\right)} .
$$

When the length of the segments is distributed with pdf $\varphi$, then 


\section{Bibliography}

\section{References}

Carroll, J. and Oldson, J. (1995). Tethers for small satellite applications. In $A I A A / U S U$ Small Satellite Conference, Logan, Utah.

Christiansen, E. L., Cykowski, E., and Ortega, J. (1993). Highy oblique impacts into thick and thin targets. International Journal of Impact Engineering, 14(1):157-168.

Cosmo, M. L. and Lorenzini, E. C., editors (1997). Tethers in space handbook, volume 4. National Aeronautics and Space Administration.

Cour-Palais, B. G. (1987). Hypervelocity impact in metals, glass and composites. International Journal of Impact Engineering, 5(1):221-237.

Flegel, S., Gelhaus, J., Wiedemann, C., Vörsmann, P., Oswald, M., Stabroth, S., Klinkrad, H., and Krag, H. (2009). The MASTER-2009 space debris environment model. In 5th European Conference on Space Debris, Darmstadt, Germany.

Francesconi, A., Giacomuzzo, C., Branz, F., and Lorenzini, E. C. (2013). Survivability to hypervelocity impacts of electrodynamic tape tethers for deorbiting spacecraft in LEO. In 6th European Conference on Space Debris, Darmstadt, Germany.

Grün, E., Zook, H., Fechtig, H., and Giese, R. (1985). Collisional balance of the meteoritic complex. Icarus, 62(2):244-272.

Hörz, F., (2012). Cratering and penetration experiments in aluminum and teflon: Implications for space-exposed surfaces. Meteoritics $\&$ Planetary Science,47(4):763-797.

Kessler, D. J., Reynolds, R. C., and Anz-Meador, P. D. (1989). Orbital debris environment for spacecraft designed to operate in low earth orbit. NASA Technical Memorandum 100-471, NASA, Lyndon B. Johnson Space Center, Houston, TX. USA.

Khan, S. B. and Sanmartín, J. R. (2013). Survival probability of round and tape tethers against debris impact. Journal of Spacecraft and Rockets, 50(3):603-608.

Khan, S. B. and Sanmartín, J. R. (2014). Analysis of tape tether survival in leo against orbital debris. Advances in Space Research, 53(9):1370-1376.

Klinkrad, H. (2006). Space Debris: Models and Risk Analysis. Berlin, Heidelberg: Praxis Publishing Ltd, Chichester, UK. 
Lambert, M (1997). Hypervelocity impacts and damage laws. Advances in Space Research, 19(2):369-378.

Liou, J.-C., Matney, M., Anz-Meador, P., Kessler, D., Jansen, M., and Theall, J. (2002). The new NASA orbital debris engineering model ORDEM2000. Technical report, National Aeronautics and Space Administration, Johnson Space Center, Houston, Texas.

NRO (1996). Tether physics and survivability (TiPS) fact sheet. Technical report, Naval Research Laboratory.

Pathria, R. K. and Beale, P. D. (1972). Statistical Mechanics, Elsevier, 1972. ISBN: 978-0-7506-2469-5

Peláez, J. and Sanjurjo, M (2006). Generator Regime of Self-Balanced Electrodynamic Bare Tethers, Journal of Spacecraft and Rockets, 43(6):1359-1369

Sanmartín, J. R., Sanchez-Torres, A., Khan, S. B., Sanchez-Arriaga, G., and Charro, M. (2015). Optimum sizing of bare-tape tethers for de-orbiting satellites at end of mission. Advances in Space Research, 56(7):1485-1492.

Sanmartín, J. R., Charro, M., Lorenzini, E. C., Garret, H. B., Bombardelli, C. and Bramanti, C. (2008). Electrodynamic Tether at Jupiter - I: Capture Operation and Constraints IEEE Transactions on Plasma Science, 36(5):2450-2458. 